\title{
Twenty Five Years After KLS: A Celebration of Non-equilibrium Statistical Mechanics
}

\author{
R.K.P. Zia
}

Received: 5 November 2009 / Accepted: 18 November 2009 / Published online: 3 December 2009

(C) Springer Science+Business Media, LLC 2009

\begin{abstract}
When Lenz proposed a simple model for phase transitions in magnetism, he couldn't have imagined that the "Ising model" was to become a jewel in field of equilibrium statistical mechanics. Its role spans the spectrum, from a good pedagogical example to a universality class in critical phenomena. A quarter century ago, Katz, Lebowitz and Spohn found a similar treasure. By introducing a seemingly trivial modification to the Ising lattice gas, they took it into the vast realms of non-equilibrium statistical mechanics. An abundant variety of unexpected behavior emerged and caught many of us by surprise. We present a brief review of some of the new insights garnered and some of the outstanding puzzles, as well as speculate on the model's role in the future of non-equilibrium statistical physics.
\end{abstract}

Keywords Non-equilibrium statistical mechanics · Driven diffusive systems · Ising lattice gas

\section{Introduction}

Over a century ago, Boltzmann and Gibbs laid the foundations for a comprehensive treatment of all systems in thermal equilibrium. By contrast, our understanding of nonequilibrium statistical mechanics (NESM) is quite primitive. To date, an overarching principle remains elusive, even for time-independent (steady) states. Yet, such systems are ubiquitous, encompassing all biological systems, for example. Thus, understanding "physics far from equilibrium" is recognized as one of the greatest challenges of current condensed matter and materials physics [1], with significant implications for technological advances in the sciences and engineering. Faced with such grand vistas, one approach is to focus on simple model systems, with the goal of identifying essential characteristics of NESM that defy our equilibrium-trained expectations and intuitions. This paper will be devoted to a seminal effort in this direction.

R.K.P. Zia ( $ه)$

Physics Department, Virginia Polytechnic Institute and State University, Blacksburg, VA 24061, USA

e-mail: rkpzia@vt.edu 
Twenty five years-and 50 Statistical Mechanics Meetings—ago, Katz, Lebowitz and Spohn (KLS) introduced a simple model [2,3], motivated partly by the physics of fast ionic conductors under the influence of an external DC field [4]. It involves a seemingly trivial modification of the two-dimensional Lenz-Ising model [5], namely, an interacting lattice gas with biased hopping along one of the axes. A brief review of this model is the main theme of this article, dedicated to the celebration of the 100th Statistical Mechanics Meeting. We will recount the many surprises it provided, the numerous variations it spawned, the aspects that are now understood, the puzzles that remain outstanding, as well as some of the general insights on NESM it offered. A more detailed review of the "first dozen years of KLS" can be found in $[6,7]$.

The KLS and the Ising models share many noteworthy features. Both represent the barest of essentials for a system with many interacting degrees of freedom to display non-trivial behavior. Each is motivated by physical systems and their interesting properties, e.g., phase transitions. In the 20's, the Ising model was "scorned or ignored" [8] as a simple mathematical toy of theorists. When Lenz proposed the model, Ising was able to solve it only in one dimension and the resultant lack of a phase transition must have disappointed theorists at the time. With the contributions of many, from Onsager [9, 10] to Wilson and Fisher [11], it became part of well-established text-book material. Not only is it one of the most celebrated models within and beyond theoretical physics, it has been realized physically in several systems [12-14]. Fortunately, the KLS model enjoyed more respect in its first decade than the Ising model did in the 30's. Unfortunately, a physical realization has yet to be found. Furthermore, it is so challenging that very little is known analytically, even in one dimension. At the "most fundamental" level, its stationary distribution is not known, in stark contrast to the explicit $\exp \left[\beta J \sum s s^{\prime}\right]$ for the Ising model in equilibrium. Consequently, most of its macroscopic properties are beyond our analytic abilities and its collective behavior, as discovered through computer simulations, continues to confound our intuitive ideas. In the remainder of this article, we briefly look back on these 25 years and look forward to much more progress on the KLS model, as well as non-equilibrium statistical mechanics in general.

\section{The Driven Lattice Gas and Its Surprising Behavior}

The KLS model is based on the Ising lattice gas $[5,15,16]$ with attractive nearest-neighbor (NN) interactions, evolving under particle-hole or spin-exchange [17] dynamics. The original study involved square lattices with periodic boundary conditions (PBC) [2, 3]. Here, let us consider a slightly more general version, with other BC's on a rectangular lattice with $L_{x} \times L_{y}$ sites (each of which may be occupied by a particle or left vacant). Thus, a configuration is specified by the occupation numbers $\left\{n_{x, y}\right\}$, where $x, y$ labels a site and $n$ is either 1 or 0 . The interparticle attraction is given by the Ising Hamiltonian: $\mathcal{H}=-4 J \sum n_{x, y} n_{x^{\prime}, y^{\prime}}$, where $x, y$ and $x^{\prime}, y^{\prime}$ are $\mathrm{NN}$ sites and $J>0$. With no drive and coupled to a thermal bath at temperature $T$, a half filled system undergoes a second order phase transition at the Onsager temperature $T_{O}=(2.2692 \ldots) \mathrm{J} / k_{B}$, from a homogeneous, disordered state to an inhomogeneous state displaying the coexistence of two regions with high and low particle densities. Minimizing surface energy, each region forms a single strip, parallel to the shorter axis. To simulate the lattice gas, a common protocol is to choose a random $\mathrm{NN}$ particle-hole pair and exchange them with probability $\min \left[1, e^{-\Delta \mathcal{H} / k_{B} T}\right]$, where $\Delta \mathcal{H}$ is the change in $\mathcal{H}$ due to the exchange.

The deceptively simple extension in KLS is to bias the particle hops along, say, the $y$ axis, so that the new rates are $\min \left[1, e^{-(\Delta \mathcal{H}+E \Delta y) / k_{B} T}\right]$. Locally, the effect of the "electric" 
field, $E$, is identical to that due to gravity. However, due to the PBC, this modification cannot be accommodated by a (single-valued) Hamiltonian. Instead, the system settles into a non-equilibrium stationary state with a non-vanishing global particle current. At first sight, this KLS model appears quite similar to the Ising case: For $T$ larger than a critical $T_{c}$, a half filled system remains in a homogeneous state, while below $T_{c}$, the system displays phase segregation. With deeper probing, dramatic differences surface at all temperatures. Moreover, many properties are entirely counterintuitive. Before discussing these surprises, let us point out that the usual Ising symmetry (particle $\Leftrightarrow$ hole, in the lattice gas language) is violated by the drive, though the combined operation of particle $\Leftrightarrow$ hole $\oplus y \Leftrightarrow-y$ (known as $\mathrm{CP}$ ) is still valid. A closely related model to KLS is the randomly driven lattice gas (RDLG), in which the sign of $E$ is chosen randomly, say, for every hop attempt [18, 19]. The effect is similar to a two-temperature Ising lattice gas (TTLG) [20-23], in which hops along $y$ are coupled to another thermal bath at temperature $T^{\prime}$ and updated with $\min \left[1, e^{-\Delta \mathcal{H} / k_{B} T^{\prime}}\right]$. For either of these, the full Ising symmetry is clearly restored. But neither will be comparable to the equilibrium Ising model and Boltzmann factors will not describe the stationary distributions of any of the driven systems.

Returning to KLS, the first surprise is how $T_{c}$ varies as $E$ is increased. For "infinite" $E$, hops aligned with the $y$ axis are accepted or rejected regardless of $\Delta \mathcal{H}$. Thus, the drive tends to break bonds, impair correlations, and increase disorder - an effect similar to being coupled to an "infinite" temperature bath. Such considerations would lead naturally to the prediction that the internal energy (average number of broken bonds) of the system should increase with $E$, so that $T_{c}$ would decrease. Simulations [2,3] showed quite the opposite: $T_{c}$ increases, saturating at $\sim 1.4 T_{O}$ for $E=\infty$ ! Remarkably, this same shift in $T_{c}$ is also observed in both the RDLG and the TTLG. Especially in the TTLG, it is natural to regard our system as coupled to two baths (with $T^{\prime}>T$ ). In this sense, we can rephrase the "first surprise" as negative response: The internal energy of our system decreases even though $T^{\prime}$ is increased. This kind of "surprising" behavior and its origins are now reasonably well understood [24], so that arguments can be advanced to predict the class of drives that would lead to increasing/decreasing $T_{c}$ 's. The general lesson here is that negative responses can be easily induced in NESM systems.

The next surprise for the early investigators is that, for all $T>T_{c}$ (where the system is homogeneous), there are long range correlations-despite the interactions and the dynamics being both short ranged. The origin of the difference between this behavior and that in the equilibrium Ising model can be traced to the violation of detailed balance and the fluctuation dissipation relation (FDR). For a system in $d$ dimensions with a conserved density, the autocorrelation function is known to decay as $t^{-d / 2}$. Given the diffusive nature for a non-critical system, the scaling $r \sim t^{-1 / 2}$ should hold and so, we should expect the equal-time correlation to decay as $r^{-d}$ [25]. The amplitude of this power turns out to be anisotropic-positive along the direction of the drive and negative otherwise, mimicking a dipolar interaction. In Fourier space, this amplitude transforms into a discontinuity singularity [26-28] of the structure factor $S(\mathbf{k})$ at $\mathbf{k}=0$, e.g., $\mathcal{R} \equiv S\left(k_{x} \rightarrow 0, k_{y}=0\right)-S\left(k_{x}=0, k_{y} \rightarrow 0\right)>0$ in $d=2$ (see also P.L. Garrido et al. and C. Maes, et al. in Ref. [20-23] and, for a summary, Ref. $[6,7])$. This behavior is "generic," in that $\mathcal{R}$ is tied to the violation of the FDR. In this sense, systems in equilibrium are "singular," since FDR forces $\mathcal{R}$ to vanish, so that the correlation in the Ising lattice gas decays as $e^{-r / \xi}$ rather than $r^{-d}$. To understand these features, it is straightforward to following the spirit of Landau-Ginzburg for the Ising model and formulate a theory for the coarse grained particle density, $\rho(\mathbf{x}, t)$. Defining $\varphi \equiv 2 \rho-1$, taking into account all anisotropies, and anticipating relevant interactions for $T<T_{c}$, we write the full 
Langevin equation

$$
\begin{aligned}
\partial_{t} \varphi(\mathbf{x}, t)= & \lambda\left\{\left(\tau_{\perp}-\nabla_{\perp}^{2}\right) \nabla_{\perp}^{2} \varphi+\left(\tau_{\|}-\alpha_{\|} \partial^{2}\right) \partial^{2} \varphi-2 \alpha_{\times} \partial^{2} \nabla_{\perp}^{2} \varphi\right. \\
& \left.+u\left(\nabla_{\perp}^{2}+\kappa \partial^{2}\right) \varphi^{3}+\mathcal{E} \partial \varphi^{2}\right\}-\xi(\mathbf{x}, t),
\end{aligned}
$$

with noise correlations

$$
\left\langle\xi(\mathbf{x}, t) \xi\left(\mathbf{x}^{\prime}, t^{\prime}\right)\right\rangle=-\left(\sigma_{\perp} \nabla_{\perp}^{2}+\sigma_{\|} \partial^{2}\right) \delta\left(x-x^{\prime}\right) \delta\left(t-t^{\prime}\right) .
$$

For the $d=2$ case, $\nabla_{\perp}$ and $\partial$ reduce to $\partial_{x}$ and $\partial_{y}$, respectively. This approach can account for all the novel properties phenomenologically, for example, with $\mathcal{R} \propto \sigma_{\perp} / \tau_{\perp}-\sigma_{\|} / \tau_{\|}$[2628] (see also P.L. Garrido et al. and C. Maes, et al. in Ref. [20-23] and, for a summary, Ref. $[6,7])$.

As $T$ is lowered towards $T_{c}$, simulations first revealed the onset of phase separation, but only transverse to the drive, corresponding to a diverging $\mathcal{R}$ but with $S\left(k_{x}=0, k_{y} \rightarrow 0\right)$ remaining finite. As a result, $k_{y}$ does not scale naively with the transverse momenta, so that analyses using techniques for "strong anisotropic scaling" are unavoidable [29, 30]. Based on these observations and starting with the Langevin equation above, a field theoretic renormalization group analysis can be set up [31-33] and, unlike the Ising universality class [34], the upper critical dimension is $d_{c}=5$. More significantly, the fixed point cannot be written in terms of a "Hamiltonian" and is genuinely "non-equilibrium" in the sense that it contains a term corresponding to a non-trivial (probability) current $[35,36]$. By contrast, a similar treatment for the RDLG leads to a fixed point Hamiltonian [18, 19], so that its leading singularities fall into the universality class of a system in equilibrium. Returning to the KLS case, a hidden symmetry associated with the fixed point is identified, so that critical exponents can be calculated to all orders in 5-d [31-33]. Thus, we expect these predictions to be reliable down to $d=2$, without the necessity of Borel resummation [34]. These novel critical properties are largely confirmed in extensive simulation studies [37-39]. Despite lingering controversies associated with claims to the contrary [40], no other field theoretic description is free of deficiencies at the basic level of symmetries [41].

Most of the surprising — and poorly understood—phenomena appear for $T<T_{c}$. Simulations showed that the ordered state is similar to the one in the Ising lattice gas: full phase separation, coexistence of a high-density region (strip) with a low-density one, interfaces aligned with the drive. Beyond these gross features in KLS, none are Ising-like and few can be predicted. Focusing on the steady state first, the most prominent feature in a phase segregated system is the interface. For $d=2$ systems in equilibrium, it is always "rough," i.e., it behaves like a random walk, with a width $w$ that scales as $L^{p}$, where $p=1 / 2$. In KLS, simulations revealed that, especially if the drive is large $(E \gg J), p$ is consistent with zero [42, 43], i.e., the interface is "smooth." Since interfaces in equilibrium systems can make transitions (the roughening transition) from being rough to smooth, a natural question is whether similar transitions exist for the KLS interface. Such an issue has never been probed systematically. Meanwhile, the roughening transition in equilibrium is associated with a singularity in the surface tension (i.e., interfacial free energy) as function of $\theta$, the angle of the interface normal. Whether a similar singularity is present for the KLS interface motivated a series of Monte Carlo studies [44] of the driven lattice gas with shifted periodic boundary conditions (SPBC), a standard technique for inducing interfaces with various normals. Figure 1(a) illustrates how "slanted" interfaces appear when a shift of 5 is imposed on a $100 \times 100$ lattice. Though there is no well-defined free energy for a system out-of-equilibrium, the internal energy $(u)$ can be easily measured and a singularity in $\theta$ will also appear in $u(\theta)$. The 
Fig. 1 (Color online) Typical configurations in a $100 \times 100$ system, driven at $E=50 \mathrm{~J}$, with $T=0.8 T_{O}$ : (a) SPBC imposed with shift 5 and (b) shift 20

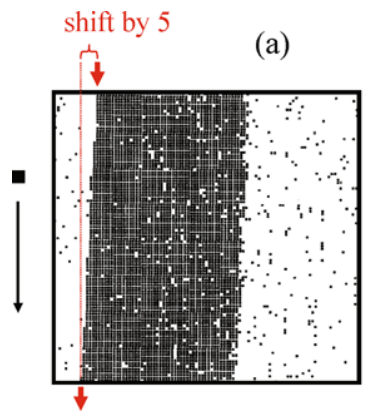

shift by 20

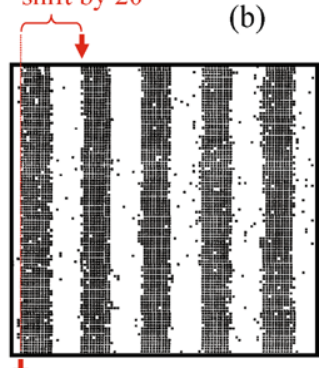

results of these studies led to the next set of surprises provided by the KLS model. First, not only is $u(\theta)$ singular $\left(\partial_{\theta} u(0)\right.$ discontinuous), it is the bulk energy density that is singular (in contrast to the Ising model with SPBC, where the bulk $u$ is independent of $\theta$ ). Further, as the shift increases, the single "slanted" strip breaks up into multiple strips, as illustrated in Fig. 1(b)! Note that the "multi-strip" configuration is actually a single strip, with multiple winding around the torus. Finally, as the shift is further increased, the system makes a series of transitions where the winding decreases one by one (" $N$ strips" merging into " $N-1$ strips")! In other words, there appears to be a series of transitions in the topology of the steady state. Though details remain to be investigated systematically, many aspects of these transitions have been explored (through simulations of relatively small systems: $72 \times 36$ ) [45]. This effort also raised further questions, as more complex phenomena emerged. For example, an attempt was made at "catching" the system at "the critical angle" where the first splitting occurs. Illustrated in Fig. 2, "icicles" (long triangular domains) appear on one of the interfaces. They are "dynamic," continuously growing and shrinking, so that the system suffers anomalously large fluctuations. From these explorations, we learn that seemingly trivial modifications of the original KLS model lead to highly complex and unexpected phenomena. Due to space limitations, we only list a few others here:

- Anomalous correlations in interfaces in both KLS and the RDLG [46, 47] ${ }^{1}$

- Steady states with "icicles" in KLS with open boundary conditions (illustrated in Fig. 3) [49]

- "Inverted icicles" during coarsening process and failures of the continuum theory [50, 51]

- Variety of pattern formation and successes of the continuum theory [52]

Most of these phenomena are far from being well understood. For example, the aspect ratio of the "icicle" pattern seems to be controlled by the microscopic parameters $(J, E, T)$, but remains to be predicted.

\section{Concluding Remarks}

In this article, we presented a very brief summary of the surprises provided by the original KLS model, i.e., an Ising lattice gas in $d=2$ with uniform and isotropic attractive interactions. Thanks to its simplicity, simulation studies were plentiful and contributed much to the

\footnotetext{
${ }^{1}$ Though there are more recent studies on the KLS interface, both the technique and the analysis are controversial [48]. It is reasonable to conclude that a definitive picture of interfacial properties remains to be established.
} 
Fig. 2 (Color online) A typical configuration in a $72 \times 36$ system with SPBC and shift 6, driven at $E=50 \mathrm{~J}$, with $T=0.8 T_{O}$. To provide a global perspective, the original (framed in a red rectangle) is reproduced multiple times in accordance with the SPBC

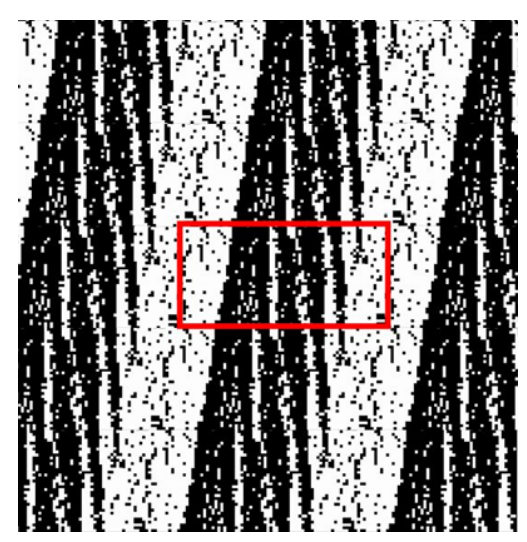

Fig. 3 A typical configuration in a $100 \times 200$ system with open boundaries, driven at $E=2 \mathrm{~J}$, with $T \simeq 0.7 T_{O}$. The top/bottom row is filled/emptied at the end of each Monte Carlo Step

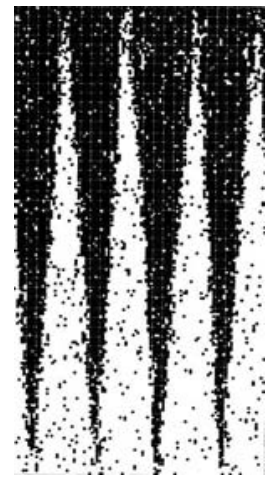

excitement associated with novel phenomena. Despite its simplicity, however, little is known analytically, not even in $d=1$ [53]. Nevertheless, we have learned a great deal from it, from specific issues such as generic long range correlations (induced by a conservation law) to a range of general properties associated with NESS. Following is a short list of the latter. (i) Negative responses should not cause a priori alarm, but should be investigated. (ii) Current loops of probability, mass, energy, etc.-whether local or global—can be expected and carry valuable information on the system. (iii) Familiar routes of the thermodynamic limit are unlikely to be reliable, while the intuition that provided successful coarse-grained continuum descriptions may lead us astray. Thus, more dependable techniques should be developed to arrive at macroscopic properties and mesoscopic theories. The overall lesson seems to be: Expect the unexpected, whenever one encounters a new NESS, no matter how trivially it appears to be related to known systems.

Although there has been only limited progress on the original KLS model, especially over the last decade, it has spawned considerable activity on several related fronts. These involve both extensions and simplifications of the original system, the subject of brief notes in the remaining paragraphs.

Extensions involve anisotropic jump rates [54, 55], anisotropic interactions [56-58] ${ }^{2}$, quenched impurities [59-62] (for another variety of quenched disorder in a driven system,

\footnotetext{
${ }^{2}$ See also Evans et al. [50, 51].
} 
see [63]), multispecies (for systems in $d=2$, see, e.g., [64-72]), ${ }^{3}$ multi-layers [73, 74], mixtures of dynamics ${ }^{4}$, to name just a few. Remarkably absent are more studies of systems in $d=3$ [75], in which new phenomena (e.g., shapes of "icicles") can be expected. Not surprisingly, since these models are more complex than KLS, even less is known analytically (except the fast rate limit $[54,55]$ ). Nonetheless, through computer simulations, these extensions provided many more surprises, especially when the modifications are so minor that no novel behavior was anticipacted! Such discoveries further challenge our basic understanding of NESS: How unpredictably complex phenomena emerge from incredibly simple dynamic rules ${ }^{5}$.

For models simpler than KLS, on the other hand, enormous advances on the theoretical front took place. One outstanding case is a lattice gas with no attractive interparticle interaction, i.e., a system of biased random walkers with on-site exclusion only. The stationary distribution becomes trivially flat for a system with PBC [76], though many interesting dynamical properties are present [77-84] (for $d=1$ cases, see, e.g., [79-84]). With open boundary conditions, even the stationary distributions are non-trivial, with few studies for systems in two or more dimensions. If we simplify further and consider one-dimensional lattices (with open boundaries), we find a wealth of analytic results. Known as the asymmetric exclusion process (ASEP) and first introduced in 1970 [76], its exact stationary state was found [85-88], showing three non-trivial phases as well as unusual dynamics [89, 90]. Also clarified is its relationship with other exactly solvable non-equilibrium systems, e.g., the zero range process [91, 92]. Furthermore, generalizations of ASEP have been exploited to model physical processes such as protein synthesis (since 1968 [93, 94](for more recent application of ASEP to protein synthesis, see, e.g. [95-103])) and vehicular traffic [104-106]. Enjoying considerable attention, it is the focus of several comprehensive reviews [107-111]. Of course, one-dimensional chains cannot support many of the interesting features discovered in the KLS, e.g., anisotropic correlations, discontinuity singularities in $S(\mathbf{k})$, and "icicles." Nevertheless, we should celebrate these contributions as giant strides towards our understanding of systems driven far from equilibrium.

To conclude, we remain hopeful that, before long, another Onsager will find an analytic solution to this amazingly rich, yet simple model, thereby shedding light on the secrets of NESS in general. Furthermore, our belief is that, like the Ising model, it will disperse its fruits far afield, beyond non-equilibrium statistical mechanics to, e.g., graph theory, quantum field theory, bioscience, neuroscience, sociophysics and econophysics.

Acknowledgements In addition to dedicating this article to the 100th Statistical Mechanics Meeting (where this material was first presented), I thank both Joel Lebowitz and Herbert Spohn for many, many illuminating discussions. The support by many collaborators, especially Beate Schmittmann, and the US National Science Foundation (through DMR-0705152) is also gratefully acknowledged.

\section{References}

1. Committee on CMMP 2010, Solid State Sciences Committee, National Research Council: CondensedMatter and Materials Physics: The Science of the World Around Us. National Academies Press, Washington (2007)

\footnotetext{
${ }^{3}$ There are many studies of 1-D systems, e.g. [70-72].

${ }^{4}$ There are many systems with mixed dynamics, especially involving spin-flip, kinetic Ising models as well. See, e.g., Sect. VII in Ref. [6, 7].

${ }^{5}$ A good example is Conway's Game of Life, made public by M. Gardner in Scientific American (October 1970).
} 
2. Katz, S., Lebowitz, J.L., Spohn, H.: Phys. Rev. B 28, 1655 (1983)

3. Katz, S., Lebowitz, J.L., Spohn, H.: J. Stat. Phys. 34, 497 (1984)

4. Chandra, S.: Superionic Solids: Principles and Applications. North Holland, Amsterdam (1981)

5. Ising, E.: Z. Phys. 31, 253 (1925)

6. Schmittmann, B., Zia, R.K.P.: In: Domb, C., Lebowitz, J.L. (eds.) Phase Transitions and Critical Phenomena, vol. 17. Academic Press, London (1995)

7. Schmittmann, B., Zia, R.K.P.: Phys. Rep. 301, 45 (1998)

8. Brush, S.G.: Rev. Mod. Phys. 39, 883 (1967)

9. Onsager, L.: Phys. Rev. 65, 117 (1944)

10. Onsager, L.: Nuovo Cimento 6, 261 (1949) (Suppl.)

11. Wilson, K.G., Fisher, M.E.: Phys. Rev. Lett. 28, 240-243 (1972)

12. Wang, G.-C., Lu, T.-M.: Phys. Rev. B 31, 5918 (1985)

13. Lynn, J.W., Clinton, T.W., Li, W.-H., Erwin, R.W., Liu, J.Z., Vandervoort, K., Shelton, R.N.: Phys. Rev. Lett. 63, 2606 (1989)

14. Wolf, W.P.: Braz. J. Phys. 30, 794 (2000)

15. Yang, C.N., Lee, T.D.: Phys. Rev. 87, 404 (1952)

16. Lee, T.D., Yang, C.N.: Phys. Rev. 87, 410 (1952)

17. Kawasaki, K.: Ann. Phys. 61, 1 (1970)

18. Schmittmann, B., Zia, R.K.P.: Phys. Rev. Lett. 66, 357 (1991)

19. Schmittmann, B.: Europhys. Lett. 24, 109 (1993)

20. Garrido, L., Lebowitz, J.L., Maes, C., Spohn, H.: Phys. Rev. A 42, 1954 (1990)

21. Maes, C., Redig, F.: J. Phys. A 24, 4359 (1991)

22. Cheng, Z., Garrido, L., Lebowitz, J.L., Vallés, J.L.: Europhys. Lett. 14, 507 (1991)

23. Praestgaard, E., Schmittmann, B., Zia, R.K.P.: Eur. Phys. J. B 18, 675 (2000)

24. Zia, R.K.P., Praestgaard, E.L., Mouritsen, O.G.: Am. J. Phys. 70, 384 (2002)

25. Grinstein, G.: J. Appl. Phys. 69, 5441 (1991)

26. Zhang, M.Q., Wang, J.-S., Lebowitz, J.L., Valles, J.L.: J. Stat. Phys. 52, 1461 (1988)

27. Hwang, K., Schmittmann, B., Zia, R.K.P.: Phys. Rev. E 48, 80 (1993)

28. Rudzinsky, M.S., Zia, R.K.P.: J. Phys. A 29, 6717 (1996)

29. Hornreich, R.M.: J. Magn. Magn. Mater. 15, 387 (1980)

30. Diehl, H.W.: Acta Phys. Slov. 52, 271 (2002)

31. Janssen, H.K., Schmittmann, B.: Z. Phys. B 64, 503 (1986)

32. Leung, K.-T., Cardy, J.L.: J. Stat. Phys. 44, 567 (1986)

33. Leung, K.-T., Cardy, J.L.: J. Stat. Phys. 45, 1087 (1986) Erratum

34. Zinn-Justin, J.: Quantum Field Theory and Critical Phenomena, 4th edn. Oxford University Press, Oxford (2002)

35. Zia, R.K.P., Schmittmann, B.: J. Phys. A 39, L407 (2006)

36. Zia, R.K.P., Schmittmann, B.: J. Stat. Mech. P07012 (2007)

37. Caracciolo, S., Gambassi, A., Gubinelli, M., Pelissetto, A.: J. Phys. A 36, L315 (2003)

38. Caracciolo, S., Gambassi, A., Gubinelli, M., Pelissetto, A.: J. Stat. Phys. 115, 281 (2004)

39. Caracciolo, S., Gambassi, A., Gubinelli, M., Pelissetto, A.: Phys. Rev. E 72, 066111 (2005)

40. Marro, J., Dickman, R.: Nonequilibrium Phase Transitions in Lattice Models. Cambridge University Press, Cambridge (1999)

41. Schmittmann, B., Janssen, H.K., Täuber, U.C., Zia, R.K.P., Leung, K.-T., Cardy, J.L.: Phys. Rev. E 61, 5977 (2000)

42. Leung, K.-T., Mon, K.K., Vallés, J.L., Zia, R.K.P.: Phys. Rev. Lett. 61, 1744 (1988)

43. Leung, K.-T., Mon, K.K., Vallés, J.L., Zia, R.K.P.: Phys. Rev. B 39, 9312 (1989)

44. Vallés, J.L., Leung, K.-T., Zia, R.K.P.: J. Stat. Phys. 56, 43 (1989)

45. Anderson, M.J.: Cooperative behavior in driven lattice systems with shifted periodic boundary conditions. Ph.D. thesis, Virginia Tech (1998). Available on-line at http://scholar.lib.vt.edu/theses/available/ etd-5598-11241/

46. Leung, K.-T., Zia, R.K.P.: J. Phys. A 26, L737 (1993)

47. Zia, R.K.P., Leung, K.-T.: J. Phys. A 24, L1399 (1991)

48. Leung, K.-T.: private communication (2009)

49. Boal, D.H., Schmittmann, B., Zia, R.K.P.: Phys. Rev. A 43, 5214 (1991)

50. Alexander, F.J., Laberge, C.A., Lebowitz, J.L., Zia, R.K.P.: J. Stat. Phys. 82, 1133 (1996)

51. Evans, M.R., Kafri, Y., Levine, E., Mukamel, D.: Phys. Rev. E 62, 7619 (2000)

52. Rutenberg, A.D., Yeung, C.: Phys. Rev. E 60, 2710 (1999)

53. Cornell, S.J., Bray, A.J.: Phys. Rev. E 54, 1153 (1996)

54. Beijeren, H., Schulman, L.S.: Phys. Rev. Lett. 86, 806 (1984)

55. Krug, J., Lebowitz, J.L., Spohn, H., Zhang, M.Q.: Phys. Rev. Lett. 44, 535 (1986) 
56. Shaw, L.B., Schmittmann, B., Zia, R.K.P.: J. Stat. Phys. 95, 981 (1999)

57. Zia, R.K.P., Shaw, L.B., Schmittmann, B.: Physica A 279, 60 (2000)

58. Levine, E., Kafri, Y., Mukamel, D.: Phys. Rev. E 64, 026105 (2001)

59. Becker, V., Janssen, H.K.: Europhys. Lett. 19, 13 (1992)

60. Lauritsen, K.B., Fogedby, H.C.: Phys. Rev. E 47, 1563 (1992)

61. Schmittmann, B., Bassler, K.E.: Phys. Rev. Lett. 77, 3581 (1996)

62. Schmittmann, B., Laberge, C.A.: Europhys. Lett. 37, 559 (1997)

63. Wu, Y., Schmittmann, B., Zia, R.K.P.: J. Stat. Mech. P04003 (2007).

64. Aertsens, M., Naudts, J.: J. Stat. Phys. 62, 609 (1990)

65. Schmittmann, B., Hwang, K., Zia, R.K.P.: Europhys. Lett. 19, 19 (1992)

66. Bassler, K.E., Schmittmann, B., Zia, R.K.P.: Europhys. Lett. 24, 115 (1993)

67. Bassler, K.E., Zia, R.K.P.: Phys. Rev. E 49, 5871 (1994)

68. Foster, D.P., Godr'eche, C.: J. Stat. Phys. 76, 1129 (1994)

69. Korniss, G., Schmittmann, B., Zia, R.K.P.: J. Stat. Phys. 86, 721 (1997)

70. Evans, M.R., Foster, D.P., Godr'eche, C., Mukamel, D.: J. Stat. Phys. 80, 69 (1995)

71. Evans, M.R., Kafri, Y., Koduvely, H.M., Mukamel, D.: Phys. Rev. E 58, 2674 (1998)

72. Clincy, M., Derrida, B., Evans, M.R.: Phys. Rev. E 67, 066115 (2003)

73. Hill, C.C., Zia, R.K.P., Schmittmann, B.: Phys. Rev. Lett. 77, 514 (1996)

74. Täuber, U.C., Schmittmann, B., Zia, R.K.P.: J. Phys. A 34, L583 (2001)

75. Marro, J., Lebowitz, J.L., Spohn, H., Kalos, M.H.: J. Stat. Phys. 38, 725 (1985)

76. Spitzer, F.: Adv. Math. 5, 246 (1970)

77. van Beijeren, H., Kutner, R., Spohn, H.: Phys. Rev. Lett. 54, 2026 (1985)

78. Janssen, H.K., Schmittmann, B.: Z. Phys. B 63, 517 (1986)

79. Dhar, D.: Phase Transit. 9, 51 (1987)

80. Gwa, L.-H., Spohn, H.: Phys. Rev. Lett. 68, 725 (1992)

81. Gwa, L.-H., Spohn, H.: Phys. Rev. A 46, 844 (1992)

82. Derrida, B., Evans, M., Mukamel, D.: J. Phys. A 26, 4911 (1993)

83. Kim, D.: Phys. Rev. E 52, 3512 (1995)

84. Derrida, B., Lebowitz, J., Speer, E.: Phys. Rev. Lett. 87, 150601 (2001)

85. Derrida, B., Domany, E., Mukamel, D.: J. Stat. Phys. 69, 667 (1992)

86. Schütz, G., Domany, E.: J. Stat. Phys. 72, 277 (1993)

87. Derrida, B., Evans, M.R., Hakim, V., Pasquier, V.: J. Phys. A 26, 1493 (1993)

88. Kolomeisky, A., Schütz, G., Kolomeisky, E., Straley, J.: J. Phys. A 31, 6911 (1998)

89. de Gier, J., Essler, F.H.L.: Phys. Rev. Lett. 95, 240601 (2005)

90. de Gier, J., Essler, F.H.L.: J. Stat. Mech. Theory Exp. P12011 (2006)

91. Evans, M.R.: Braz. J. Phys. 30, 42 (2000)

92. Evans, M.R., Hanney, T.: J. Phys. A 38, R195 (2005)

93. MacDonald, C.T., Gibbs, J.H., Pipkin, A.C.: Biopolymers 6, 1 (1968)

94. MacDonald, C.T., Gibbs, J.H.: Biopolymers 7, 707 (1969)

95. Shaw, L.B., Zia, R.K.P., Lee, K.H.: Phys. Rev. E 68, 021910 (2003)

96. Lakatos, G., Chou, T.: J. Phys. A 36, 2027 (2003)

97. Shaw, L.B., Sethna, J.P., Lee, K.H.: Phys. Rev. E 70, 021901 (2004)

98. Chou, T., Lakatos, G.: Phys. Rev. Lett. 93, 198101 (2004)

99. Dong, J.J., Schmittmann, B., Zia, R.K.P.: J. Stat. Phys. 128, 21 (2007)

100. Dong, J.J., Schmittmann, B., Zia, R.K.P.: Phys. Rev. E 76, 051113 (2007)

101. Dong, J.J., Zia, R.K.P., Schmittmann, B.: J. Phys. A 42, 015002 (2009)

102. Cook, L.J., Zia, R.K.P.: J. Stat. Mech. Theory Exp. P02012 (2009)

103. Cook, L.J., Zia, R.K.P., Schmittmann, B.: Phys. Rev. E 80, 031142 (2009)

104. Nagel, K., Schreckenberg, M.: J. Phys. 12, 2221 (1992)

105. Chowdhury, D., Santen, L., Schadschneider, A.: Phys. Rep. 329, 199 (2000)

106. Schmittmann, B., Krometis, J., Zia, R.K.P.: Europhys. Lett. 70, 299 (2005)

107. Schütz, G.M.: In: Domb, C., Lebowitz, J.L. (eds.) Phase Transitions and Critical Phenomena, vol. 19. Academic Press, New York (2000)

108. Spohn, H.: Physica A 369, 77 (2006)

109. Derrida, B.: J. Stat. Mech. P07023 (2007)

110. Sasamoto, T.: J. Stat. Mech. P07007 (2007)

111. Blythe, R.A., Evans, M.R.: J. Phys. A 40, R333 (2007) 